\title{
Using the 3-D structures of the viral proteinases of Porcine Epidemic Diarrhoea Virus (PEDV) to design anti-PEDV drugs
}

Tooba Naz Shamsi, Jiang Yin, Michael James

Department of Biochemistry, Faculty of Medicine and Dentistry, University of Alberta, Edmonton, Canada

tshamsi@ualberta.ca

The Porcine epidemic diarrhea virus (PEDV) is a coronavirus that causes severe diarrhoea and a high mortality rate in suckling piglets. Traditional antiviral measures such as vaccinations have not shown sufficient effectiveness in protecting piglets against PEDV infection. Given its important role in viral polyprotein processing, the PEDV $3 \mathrm{C}$-like proteinase $\left(3 \mathrm{CL}^{\text {pro }}\right)$ may be a potential target for the development of antiviral drugs adopting a structure-guided approach. To facilitate these efforts, we have expressed, purified, and successfully crystallized a recombinant PEDV 3CL $L^{\text {pro }}$ that presents the cognate $\mathrm{N}$ - and C-termini of the viral protein. In addition, we have developed an in vitro enzymatic activity assay for screening anti-PEDV 3CL ${ }^{\text {pro }}$ inhibitors using FRET-based peptide substrates. We are currently working to solve the 3-dimenstional structures of PEDV $3 \mathrm{CL}^{\text {pro }}$ in complex with various substrate analogues and inhibitors. The structural information garnered through these studies will help shed light on the mechanisms of catalysis and inhibition as well as possible chemical modifications of the inhibitors that may improve their specificity and potency. 